\title{
Charting Terra Incognita at Alto and S3
}

\author{
Serge Franchoo ${ }^{1, \star}$, Maher Cheikh Mhamed ${ }^{1}$, Ruohong $\mathrm{Li}^{1, \star \star}$, Enrique Minaya Ramirez ${ }^{1}$, Liss \\ Vazquez-Rodriguez ${ }^{1}$, and Deyan Yordanov ${ }^{1}$ \\ ${ }^{1}$ IPN Orsay, France
}

\begin{abstract}
Charting Terra Incognita is a project grant that brings together the IPN Orsay, CSNSM Orsay and Irfu-SPHN laboratories around the common development of knowhow, equipment and techniques for mass spectrometry and nuclear spectroscopy at the low-energy branch of the new S3 spectrometer at Ganil, where likewise the Reglis set-up for intrajet laser spectroscopy is entering its final phase of construction. At the same time its concerted action initiates and sets the pace for the corresponding precursor physics programme at the Alto on-line isotope-separation facility, based on the photofission of uranium. We also collect a review of the Alto laser ion source, which is an essential instrument at any low-energy facility for the production of purified isotopic beams.
\end{abstract}

\section{Introduction}

Alto is an infrastructure that comprises an on-line isotope-separation facility based on the photofission of uranium next to a stable ion beam facility based on a 14.5-MV tandem accelerator. The isotopeseparation on-line section of Alto is dedicated to the production of neutron-rich radioactive ion beams (RIB) from the interaction of the $\gamma$ flux induced by a 500-W electron beam in a uranium carbide target. It is dimensioned for $10^{11}$ fissions per second. Its laser ion source has so far delivered radioactive beams of gallium and zinc isotopes, while indium and tin have been ionised off-line. After mass separation, the RIBs are presently sent to an experimental beam line dedicated to $\beta$ decay, while the construction of three set-ups for low-temperature nuclear orientation, collinear laser spectroscopy and a double Penning trap for mass measurements is in progress. The RIB facility is exploited in alternating mode with the tandem machine, capable of accelerating light ions for nuclear astrophysics and heavy ions for $\gamma$ spectroscopy. The facility thereby offers the opportunity to provide beams to a large range of physics programmes from nuclear to interdisciplinary physics. In this contribution the laser ion source of Alto is presented as well as an overview given of the three new installations. We discuss the prospects for their experimental programme, which is initiated at Alto today and will later on be continued at the S3 spectrometer at Ganil.

\section{The Rialto resonant laser ion source}

The Alto linear accelerator delivers $10 \mu \mathrm{A}$ of electrons at an energy of $50 \mathrm{MeV}$. It was recovered from Cern, where it served as the injector of the Large Electron-Positron Collider till the latter was

\footnotetext{
$\star$ e-mail: franchoo@ipno.in2p3.fr

$\star \star$ Present address: Triumf, Vancouver, Canada
} 
dismantled in 2001. After the validation of its potential for photofission, the accelerator was moved to IPN Orsay and reassembled in the main experimental area that also houses the Parrne mass separator [1]. It was put into operation in December 2005 and the first photofission production runs on a uranium carbide target took place in June 2006. It is the bremsstrahlung of the primary electron beam when it penetrates and slows down in the first centimeters of the thick target that provokes the photofission of the surrounding uranium nuclei. The fission fragments are separated by mass on-line and sent to the detection station. Alto thereby opens a window to study neutron-rich nuclei at competitive rates at a relatively low investment cost.

In order to increase the selectivity of the beam extraction, it was soon decided to equip the facility with a laser ion source. A standard ion source used for both surface and laser ionisation consists essentially of a target that is coupled to an ionisation tube. The tube is most often made of tantalum but sometimes tungsten and kept at an elevated temperature by ohmic heating. While an exact temperature calibration is difficult because of the changing environment induced by heating and irradiation, a reference measurement shows that increasing the current of a tantalum tube under typical conditions from 170 A to 320 A raises the temperature from 650 to $1900{ }^{\circ} \mathrm{C}$. A separate receptacle is connected to the ionisation tube by means of a small duct and contains a sample of the material of interest. For this mass marker we originally used about $100 \mathrm{mg}$ of metallic powder, which after a period of initial tuning of the ion source would drop to some $10 \mathrm{nA}$ for the laser measurements to begin. In the most recent experiments we replaced the powder with salt solutions of the order of $100 \mu \mathrm{g}$, allowing us to achieve an evaporation rate that is more controllable and reproducible. The design of an ion source for stable beams is identical but does not include the target.

When heated only, the tube acts as a surface ioniser for elements with a low work function. If laser light at suitable wavelengths is sent into the cavity, also element-selective resonant ionisation takes place. This can be ensured by a laser system with high repetition rate. At Alto, monochrome radiation at $532 \mathrm{~nm}$ from a 100-W Nd:Yag laser purchased from Edgewave pumps three tunable dye lasers at a rate of $10 \mathrm{kHz}$. The ranges of the two Narrowscan dye lasers from Radiant Dyes nominally span from 540 to $900 \mathrm{~nm}$ and of the third Liopstar-HQ model from Lioptec from 430 to $900 \mathrm{~nm}$. Each is equipped with a BBO crystal for frequency doubling and four Pellin-Broca prisms for separating the desired component. The laser ionisation schemes are taken from the Isolde database $[2,3]$.

The first element that was resonantly ionised at Alto was gallium. Rhodamine $6 \mathrm{G}$ dissolved in ethanol was used to produce ultraviolet light at $287.5 \mathrm{~nm}$ after frequency doubling. This excites the $4 s^{2} 4 p^{2} P_{1 / 2}$ ground state to a $4 s^{2} 4 d^{2} D_{3 / 2}$ state at $34782 \mathrm{~cm}^{-1}$. Ionisation into the continuum took place with the Nd:Yag laser light at $532 \mathrm{~nm}$. With $360 \mathrm{~mW}$ of 287.5-nm ultraviolet power, $21 \mathrm{~W}$ of Nd:Yag power, and transport efficiencies for the two laser beams to the ion source 12 meter away of 55 and $65 \%$ respectively, we obtained 2 to $7 \mathrm{nA}$ of ${ }^{69} \mathrm{Ga}^{+}$above a non-resonant background of 2 $\mathrm{pA}$ with a current on the ionisation tube of $170 \mathrm{~A}$. When the ioniser current was increased to 200 $\mathrm{A}$, the gallium signal went up to $17 \mathrm{nA}$ for the same background rate. At $250 \mathrm{~A}$, we measured 70 $\mathrm{nA}$ of gallium but with a background that rose to $45 \mathrm{pA}$. Finally at $320 \mathrm{~A}$, we started depleting the gallium sample, the current of which dropped to $28 \mathrm{nA}$, while the background climbed to $2 \mathrm{nA}$. This enhancement of the ion rate of a factor of 14 was also observed for radioactive ${ }^{83} \mathrm{Ga}$.

In a second experiment, again at $320 \mathrm{~A}$ of ioniser current and with transport efficiencies that ranged from 41 to $56 \%$, an enhancement of 40 was obtained for stable mass-separated gallium, but for the radioactive beam this factor fell to 2 . A possible explanation might be found in the condition of the source, which was saturated by stable isotopes from the mass marker. Because of their overwhelming number, these would absorb most of the available photons.

At a temperature of $2000{ }^{\circ} \mathrm{C}, 54 \%$ of the particles reside in a ${ }^{2} P_{3 / 2}$ metastable state at 826 $\mathrm{cm}^{-1}$ above the ground state. This metastable state was accessed at $294.5 \mathrm{~nm}$, for which we used pyrromethene 597 in ethanol. The efficiency of the dye rapidly dropped, however, losing $20 \%$ of its 
peak value within four hours. In the second experiment this short lifetime was prolonged by adding diazabicyclo-octane (also known as dabco), which acts as an anti-oxydant. With $270 \mathrm{~mW}$ of $287.5-\mathrm{nm}$ and $130 \mathrm{~mW}$ of $294.5-\mathrm{nm}$ ultraviolet power, the laser enhancement could be worked up from a factor that varied between 2 and 3 up to a value between 5 and 7 .

Zinc was ionised in a three-step scheme. DCM was dissolved in ethanol to create 213.9-nm ultraviolet radiation after frequency tripling. The $4 s^{2}{ }^{1} S_{0}$ ground state is lifted to a $4 s 4 p{ }^{1} P_{1}$ state at $46745 \mathrm{~cm}^{-1}$. The second step at $636.4 \mathrm{~nm}$ was also provided by DCM in ethanol, bringing the atom to a $4 s 4 d{ }^{1} D_{2}$ level at $62459 \mathrm{~cm}^{-1}$. The Nd:Yag laser at $532 \mathrm{~nm}$ assured the ionisation into the continuum. Laser powers up to $36 \mathrm{~mW}$ of far ultraviolet and $3.6 \mathrm{~W}$ of red and were obtained, with between 16 and $20 \mathrm{~W}$ left for the Nd:Yag final transition. Transport efficiencies up to 39 and $48 \%$ were measured for the red and Nd:Yag beams respectively. Saturation was reached for the red transition only. At an ioniser current of $280 \mathrm{~A}$, a resonant signal of stable ${ }^{64} \mathrm{Zn}^{+}$of $3 \mathrm{nA}$ was found above a background of $5 \mathrm{pA}$, rising to $5 \mathrm{nA}$ at a current of $320 \mathrm{~A}$. Radioactive ${ }^{76,79,80} \mathrm{Zn}$ were laser ionised.

For a second experiment $22 \mathrm{~mW}$ of the ultraviolet tripled transition and $2.0 \mathrm{~W}$ of the red firstharmonic light were produced, while $33 \mathrm{~W}$ were made available for the Nd:Yag ionising transition. With 330 A of current on the ionisation tube, we observed $3 \mathrm{nA}$ of stable zinc on a $3 \mathrm{pA}$ background. The heating of the ioniser was then increased to $380 \mathrm{~A}$ to hasten the release of radioactive ${ }^{74-79} \mathrm{Zn}$, which were laser ionised albeit at a production rate of a factor of 6 below the first experiment.

If one takes a close look at the behaviour of the production rates as a function of the isotopic number and compares these to the expected yields for photofission at Alto [4, 5], accounting for the release from the target with a 5.40-s time constant [6] and assuming an efficiency of laser ionisation of 5\% [2] and normalising to the better rates of the first experiment, one finds a residual efficiency of $4.6 \%$ for ${ }^{76} \mathrm{Zn}$ and ${ }^{78} \mathrm{Zn}$. The missing factor of 20 likely is to be ascribed to the various target and ion source parameters and in principle is recoverable once sufficient resources and effort are dedicated to investigating these and lining the target and ion source up to the performance of the well studied system at Isolde, of which it remains a faithful copy.

Normalising on the measurement for ${ }^{76} \mathrm{Zn}$ and ${ }^{78} \mathrm{Zn}$, nevertheless one observes that the yield drops off faster than foreseen for the most exotic isotopes. From ${ }^{77} \mathrm{Zn}$ to ${ }^{78} \mathrm{Zn}$ we discern a decrease by a factor of 3.4 whereas from cross-section calculations with release-time and half-life corrections we had forecast 1.6. From ${ }^{78} \mathrm{Zn}$ to ${ }^{79} \mathrm{Zn}$ we go down by 5.6 while we had anticipated 1.8 . The situation is reminiscent of the gallium case, for which we mentioned above the lower efficiency for the radioactive beam. Two lines of reasoning can be offered for this effect. Firstly, charge-exchange processes are prone to neutralise the ions of interest when these collide with stable atoms that are present in the source. In particular if the contaminant is an alkali metal such as rubidium, which is often added to the ion source at Alto for monitoring the beam transport, it will readily cede an electron to the laser ion. The charge-exchange probability would be the same for all isotopes of zinc but it would imply that larger groups of not-so-exotic zinc possibly stand a bigger chance of survival than smaller pockets of exotic zinc. Secondly, when a small quantity of radioactive atoms is submerged within a vast amount of stable atoms of the same species, the stable atoms may consume a certain fraction of the flow of laser photons before there is a chance for the radioactive ones to do so. Such large amounts of stable isotopes can for instance originate from the evaporation of a mass marker in an uncontrolled manner. The combination of both effects may then explain the low efficiency for the most exotic isotopes. It shows the need for mass markers, both alkalis for use in beam transport as isotopes for laser optimisation, that can be heated independently and switched off when no longer required. In such a way one may prevent the ion source from being overloaded with stable particles when weaker radioactivities are to be measured. Such a development, based on the know-how from Isolde, is currently being implemented at Alto. 
Also tin was laser-ionised in a three-step scheme. In a first attempt a metastable state at an energy of $1692 \mathrm{~cm}^{-1}$ was selected. Ultraviolet light was generated at $301.0 \mathrm{~nm}$ with $200 \mathrm{~mW}$ of power, of which $63 \%$ could be transported to the ion source. For the dye we chose a mixture of $80 \%$ of Rhodamine B and 20\% of Rhodamine 101 dissolved in methanol. The lifetime of this solution was of the order of one week. The $5 p^{2}{ }^{3} P_{1}$ state could thus be excited to a $5 p 6 s^{3} P 1$ level at $34914 \mathrm{~cm}^{-1}$. With Styryl 9 in ethanol providing two infrared lasers of $811.6 \mathrm{~nm}$ and $823.7 \mathrm{~nm}$, the latter level was first raised to a $5 p 6 p^{3} P_{2}$ structure at $47235 \mathrm{~cm}^{-1}$ and then to a $5 p 9 s^{3} P_{2}$ auto-ionising state. These two lasers provided rather modest powers of 270 and $530 \mathrm{~mW}$ with transport ratios to the ion source of 27 and $35 \%$, respectively, since the transitions were expected to stand closer to saturation than the UV one. A laser enhancement of 10, being $70 \mathrm{nA}$ of signal over $7 \mathrm{nA}$ of background, was observed for stable ${ }^{120} \mathrm{Sn}$ at an ioniser current of $320 \mathrm{~A}$. When the non-resonant radiation of the $\mathrm{Nd}$ :Yag replaced the 823.7-nm transition, we found $40 \mathrm{nA}$ of laser-ionised tin over a background of $8 \mathrm{nA}$. The reason for the surprisingly high background current, which was already noticed in the case of zinc, is not known but could point to difficulties related with the temperature calibration of the ion source.

The choice of the metastable state made it possible to ionise also indium with the same laser setup. Advantage was taken of the ultraviolet transition at $304.0 \mathrm{~nm}$, which could be generated with the dye we mentioned also at a power of $200 \mathrm{~mW}$, from the $5 p^{2} P_{1 / 2}$ ground state to a $5 d^{2} D_{3 / 2}$ level at $32892 \mathrm{~cm}^{-1}$, followed by the 532-nm Nd:Yag radiation. The enhancement factor for stable ${ }^{115} \mathrm{In}$ only amounted to 1.5 , yielding $3.2 \mathrm{nA}$ of signal over $2.1 \mathrm{nA}$ of background. The low increase might be due to the use of an ionisation tube of tungsten still held at $320 \mathrm{~A}$, creating an environment that was sufficiently hot for a strong current of surface-ionised stable indium to develop. Relative to this non-resonant current, the laser ions do not stand out as clearly as they would when the background is suppressed at low temperature. A similar situation was encountered in the case of gallium that we described above. Indeed when most of the sample had evaporated, the signal became more evident with $130 \mathrm{pA}$ of laser-ionised indium over $50 \mathrm{pA}$ of background.

The ionisation of tin was further studied in an off-line evaporation chamber, which was designed with help from the university of Mainz in Germany. The chamber contains a graphite oven that can reach $2100{ }^{\circ} \mathrm{C}$. Mass separation is not available in this set-up. To generate the tin vapour, several drops of a $\mathrm{SnCl}_{2}$ salt solution were deposited on a tantalum foil of $1 \mathrm{~cm}^{2}$ that was rolled up, inserted in the oven and heated to $930{ }^{\circ} \mathrm{C}$. This time the $5 p^{2}{ }^{3} P_{0}$ ground state of tin was taken and excited by a 286.4-nm ultraviolet photon, produced from Rhodamine $6 \mathrm{G}$ in ethanol with $160 \mathrm{~mW}$ of power. The first and second infrared lasers now stood at values up to $1.2 \mathrm{~W}$ and $2.7 \mathrm{~W}$, respectively. The ionised particles were guided with a set of three electrostatic lenses to a secondary-electron multiplier. Since the temperature was kept low compared to the set-up at the on-line mass separator, the background that originates from surface ionisation could be reduced. With a small positive voltage the remainder was repelled and a background-free signal of $4 \mathrm{nA}$ could be sustained. It confirms that the full potential of resonant laser ionisation can be recognised only under well controlled conditions of temperature and extraction fields.

While a quest for increased reliability and remote control remains the priority for the laser ion source at Alto, next avenues may consist in the modelisation of the off-line chamber in order to determine the ionisation efficiency quantitatively, or the implementation of automated scanning, for which a better understanding of the background conditions is required.

\section{Terra Incognita of exotic nuclei at Alto at S3}

One of the main goals of modern nuclear physics is the study of exotic radioactive nuclei, addressing questions such as the limit of existence for extreme neutron to proton ratios. The available data become scarce as one ventures far from stability and obtaining the first information on ground-state 


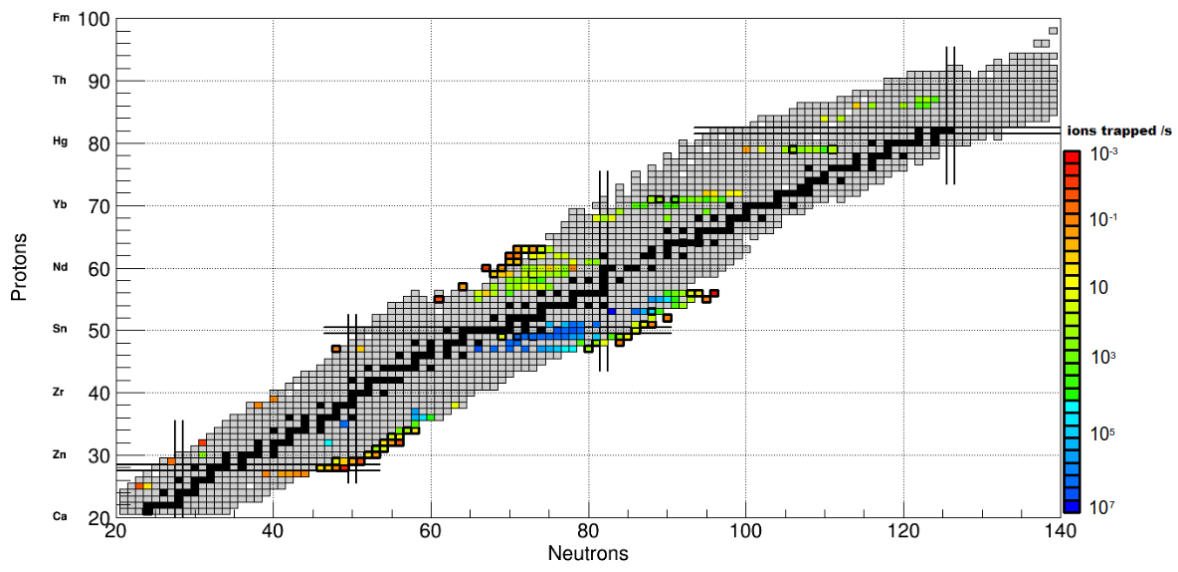

Figure 1. Nuclides accessible for mass measurements in photofission and fusion evaporation at Alto $\left(t_{1 / 2}>80\right.$ ms, $\delta m>10 \mathrm{keV}$, ions trapped per second). Nuclides not experimentally accessed by mass spectrometry so far are marked with a thick border

properties such as nuclear mass and radius provides a important step in better understanding the force that binds the protons and neutrons together inside the nucleus, how the nucleus behaves when neutrons and protons occupy various quantum states, when the Coulomb repulsion between protons becomes very large or when the outer neutrons extend in space well beyond the core.

The low production cross sections of those nuclei and the presence of dominant contributions from contaminating reaction channels, however, make them often difficult to study. At Alto, the front-end is configured to receive different driver beams, being electrons for photofission of uranium carbide and heavy ion for fusion-evaporation reactions in suitable, medium-mass targets. Photofission introduces as little excitation energy as possible into the fissioning nucleus and therefore it is essentially a cold process, which produces exotic neutron-rich species that are of relatively high isotopic and isobaric purity. For the remaining sample, the element selectivity of resonant laser ionisation that was described in the previous section is coupled to the mass selectivity of electromagnetic separation to select the nuclei of interest, without being compromised by the contaminations that may be present in large numbers at other facilities. Masses, moments and other ground-state properties can then be accurately measured.

Funding has recently been acquired for the installation of three detection set-ups Polarex, MLLtrap, and Lino. Presently in different stages of development, they are being designed for, or adapted to, high-resolution measurements of the radioactive ions that will be produced at Alto. At first, the physics programme will cover the fission products that are already available at Alto. In a next stage the possibility of a fusion-evaporation ion source will be investigated, giving access to neutron-deficient nuclei above doubly magic ${ }^{100} \mathrm{Sn}$. A nuclear chart with the nuclides that are expected to be accessible in photofission and fusion-evaporation reactions for high-precision mass measurements at Alto is shown in figure 1.

Polarex is a ${ }^{3} \mathrm{He}-{ }^{4} \mathrm{He}$ dilution refrigerator formerly owned by Triumf in Canada and reassembled at CSNSM in Orsay [7]. It uses nuclear orientation at low temperatures below $15 \mathrm{mK}$ to study exotic nuclei implanted in a ferromagnetic host of 10 to $100 \mathrm{~T}$. The implantation foil itself is magnetised through an externally applied field of $1.5 \mathrm{~T}$. Under these conditions the nuclei turn into a polarised ensemble that may exhibit remarkable properties, which are of interest to various research fields such as 
solid-state physics, fundamental-interaction studies and nuclear structure. From the angular distribution of the radiation emission of the sample, magnetic moments and spins can be measured as well as finer observables such as $\gamma$-multipolarity ratios and parity admixtures after $\beta$ decay. The physics case centres on magnetic properties close to doubly magic ${ }^{132} \mathrm{Sn}$. For instance an abrupt change in the level structure has been observed in ${ }^{135} \mathrm{Sb}$ and imputed to the migration of single-particle orbitals [8]. The same effect can be predicted for ${ }^{136,137} \mathrm{I}$ and should be apparent from the magnetic moments. Since the beam line is connected from below, a large number of detectors can easily be mounted around the sample resulting in a good spatial resolution of the anisotropy. The recording of subsequent $\gamma$ or $\beta n$ decay will then add to the versatility of the experiment.

MLLtrap is a double Penning trap that was constructed at the Maier-Leibnitz Laboratory (MLL) in Munich in Germany [9]. It was transported and is now being installed and commissioned at Alto in Orsay. The phase-imaging ion-cyclotron resonance detection technique will be implemented in order to perform high-precision mass measurements of short-lived nuclides that are produced at low rates [10]. In particular masses of exotic nuclei in the region of $A=130$ are crucial to understand the evolution of the shell gap at $N=82$. The strength of shell closures gives constraints on the description of the nuclear force, but also influences the path of the $r$-process and the predictions of elemental abundances. Knowledge of the masses of neutron-rich ${ }^{121-128} \mathrm{Ag}$ and ${ }^{128-134} \mathrm{In}$ would allow to investigate a possible weakening of the shell gap below tin and its impact on the $A=130 r$-process nucleosynthesis.

We shall also exploit the carrier-free storage capabilities of the ion trap for validating a proof of principle of particle-decay spectroscopy in the trap. In particular a trapped heavy ion may first emit an $\alpha$ particle, which is then followed by a conversion electron. Shake-off and Auger electrons that are generated in these processes will be magnified by the magnetic field of $7 \mathrm{~T}$ in the trap and focussed on position-sensitive silicon detectors that replace the trapping electrodes. From the distance the two electron clouds develop, the lifetime of the nuclear excited state can be determined. One estimates an effect of $50 \mu \mathrm{m}$ to $1.5 \mathrm{~mm}$ for a half-life of $100 \mathrm{ps}$. At Alto emphasis will be put on establishing the proper procedures that are needed for performing such measurements without energy loss nor summing effects.

The scientific overlap between mass spectrometry and laser spectroscopy consists in the complementary picture of the nuclear ground state that is obtained. With the Lino laser beam line, for which a 15-W pump laser at $532 \mathrm{~nm}$ followed by a Ti:Sa ring laser and a frequency-doubling CW wavetrain is envisaged, atomic isotope shifts and hyperfine structures will initially be measured, from which nuclear charge radii, ground-state spins and electromagnetic moments can be extracted or confined. In addition both techniques are capable of discovering and probing nuclear isomers, enabling a comprehensive characterisation of the nuclear states by laser spectroscopy and determining their energy order from mass measurements. In a next development that Lino proposes, the polarisation of the laser light will be transferred to the ions, such one becomes sensitive to the $\beta$ asymmetry parameters. The reach of Lino will then be expanded to direct measurements of the spins of excited states by $\beta$-delayed spectroscopy. Lino shall typically be dedicated to isotopes that are produced at rates below $10^{4}$ particles per second and with half-lives shorter than one second, beyond what Polarex can access. The synergy between the two methods will also be explored in terms of low-energy beam manipulation and handling, namely the installation of radio-frequency quadrupole Paul traps adapted to the requirements of either technique. The measurement programme intends to set off also here with the nuclei around tin, which are excellent probes for nuclear structure due to the influence of the shell closures at $Z=50$ and $N=82$ and at the same time key ingredients in understanding stellar nucleosynthesis. The available data away from the valley of stability remain insufficient and apart from the study of cadmium [11] there has been no experimental output in the recent past. At Alto a long sequence of 16 isotopes between ${ }^{111} \mathrm{Ag}$ and ${ }^{126} \mathrm{Ag}$ is accessible, most of which have not been studied conclusively 


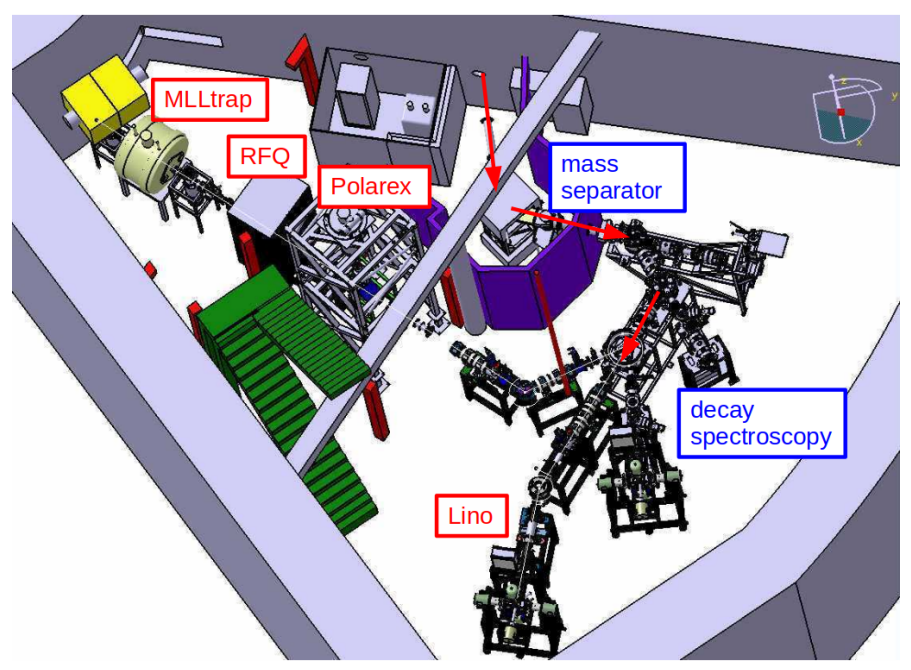

Figure 2. Lay-out of the Alto experimental area with the Polarex, MLLtrap and Lino set-ups

until now. Silver moreover is an example of nuclear isomerism, the longer-lived states of which are accessible to laser spectroscopy. Further onwards the indium and antimony chains will be aimed at.

The new S3 spectrometer at Ganil will allow for the continuation and extension of the physics programme of MLLtrap, as ${ }^{100} \mathrm{Sn}$ will become within reach and superheavy elements will be produced in sufficient amounts for detailed studies [12]. Advantage will be taken from the experience developed at Alto to broaden the intratrap measurements to $\alpha$ and conversion-electron decay. Laser spectroscopy will instead be performed with Reglis, which is based on a gas cell filled with argon at 350 mbar of pressure that will stop and neutralise the reaction products from S3 [13]. With a dedicated optical mode that optimised transmission instead of resolution, rates at the final focal plane of $310^{4}$ particles per second for nuclei such as ${ }^{152} \mathrm{Er}$ and 10 per second for ${ }^{254} \mathrm{No}$ are currently anticipated, spread over several charge states within a beam area of $50 \mathrm{~mm}$ in diameter. The gas flow then carries the particles along towards the exit hole of the gas cell, where a carefully controlled gas jet develops for which the pressure falls below 1 mbar. For ${ }^{215} \mathrm{Ac}$ as a test case, it has been shown that ionisation in the supersonic jet can reach a resolution of $400 \mathrm{MHz}$, while room for improvement down to $100 \mathrm{MHz}$ is projected by increasing the Mach number from 6 to 10 [14]. A sequence of radiofrequency ion guides and a quadrupole mass filter finally will prepare an ion beam at low emittance that can be sent towards other experiments.

\section{Acknowledgments}

We are grateful for the support we received from T. Day Goodacre, V. Fedosseev and B. Marsh from Isolde, Switzerland, and K. Flanagan from the university of Manchester, United Kingdom, in the set-up of the laser ion source. We also thank T. Kron and K. Wendt from the university of Mainz, Germany, for their guidance in the construction of the off-line evaporation chamber. Charting Terra Incognita of Exotic Nuclei is a collaboration between IPN Orsay, CSNSM Orsay and Irfu-SPHN that is selected for financial support as a emblematic project by the laboratory of excellence Labex P2IO under tutorship of CEA, CNRS, Ecole Polytechnique and the university of Paris-South. We thank A. Drouart, C. Gaulard, A. Lopez-Martens, L. Perrot, S. Roccia, A Said and B. Sulignano for their 
contributions, without which the project would not be possible. Reglis is funded by a grant from the National Research Agency of France under contract number ANR-13-BS05-0013 attributed to IPN Orsay, Ganil, LPC Caen and the university of Louvain in Belgium.

\section{References}

[1] F. Ibrahim et al., Eur. Phys. J. A 15, 357 (2002)

[2] V. Fedosseev et al., Nucl. Instr. and Meth. in Phys. Res. B 266, 4378 (2008)

[3] V. Fedosseev, Y. Kudryavtsev and V. Mishin, Physica Scripta 85, 058104 (2012)

[4] F. Ibrahim et al., Ninth International Conference on Nucleus-Nucleus Collisions, Rio de Janeiro, Brazil, 2006, Nucl. Phys. A 787, 110 (2007)

[5] M. Cheikh Mhamed et al., 15th International Conference on Electromagnetic Isotope Separators and Techniques Related to their Applications, Deauville, France, 2007, Nuclear Instr. and Meth. B 266, 4092 (2008)

[6] B. Roussière, private communication (2015)

[7] L. Risegari et al., European Physical Journal A 42, 307 (2009)

[8] J. Shergur et al., Phys. Rev. C 65, 034313 (2002)

[9] C. Weber, P. Müller, and P. Thirolf, Int. J. of Mass Spectrometry 349, 270 (2013)

[10] S. Eliseev et al., Phys. Rev. Lett. 110, 082501 (2013)

[11] D. Yordanov et al., Phys. Rev. Lett. 110, 192501 (2013)

[12] A. Drouart et al., Nucl. Phys. A 834, 747 (2010)

[13] R. Ferrer et al., Nucl. Instr. and Meth. in Phys. Res. B 317, 570 (2013)

[14] R. Ferrer et al., Nature Communications 8, 14520 (2017) 